\title{
AN UNCONDITIONALLY STABLE NONSTANDARD FINITE DIFFERENCE METHOD APPLIED TO A MATHEMATICAL MODEL OF HIV INFECTION
}

\author{
HASIM A. OBAID ${ }^{*}$, RACHID OUIFKI ${ }^{* *}$, KAILASh C. PATIDAR ${ }^{* * *}$ \\ * Department of Mathematics and Applied Mathematics \\ University of the Western Cape, Bellville 7535, South Africa \\ **DST/NRF Centre of Excellence in Epidemiological Modelling and Analysis \\ Stellenbosch University, Stellenbosch 7600, South Africa \\ *** Department of Mathematics and Applied Mathematics \\ University of the Western Cape, Private Bag X17, Bellville 7535, South Africa \\ e-mail: kpatidar@uwc.ac.za
}

\begin{abstract}
We formulate and analyze an unconditionally stable nonstandard finite difference method for a mathematical model of HIV transmission dynamics. The dynamics of this model are studied using the qualitative theory of dynamical systems. These qualitative features of the continuous model are preserved by the numerical method that we propose in this paper. This method also preserves the positivity of the solution, which is one of the essential requirements when modeling epidemic diseases. Robust numerical results confirming theoretical investigations are provided. Comparisons are also made with the other conventional approaches that are routinely used for such problems.
\end{abstract}

Keywords: HIV infection, dynamical systems, nonstandard finite difference methods, equilibria, stability.

\section{Introduction}

Mathematical models play a significant role in understanding the dynamics of biological systems. In most cases, these models are described by autonomous systems of nonlinear ordinary differential equations, (see, for example, the works of Kouche and Ainseba (2010), Xu et al. (2011), and the references therein). Very often, such systems are so complex that their exact solutions are not obtainable and hence the need for robust numerical methods arises. However, as mentioned by Villanueva et al. (2008), numerical methods like those of Euler, Runge-Kutta and others fail to solve nonlinear systems generating oscillations, chaos, and false steady states. One way to avoid such numerical instabilities is the construction of nonstandard finite difference schemes. Dimitrov and Kojouharov (2007) pointed out that numerical methods one uses to approximate the solutions of dynamical systems are expected to be consistent with the original differential systems, and should be zero-stable and convergent.

In view of the above, in this paper we design a special class of numerical methods, known as NonStandard
Finite Difference Methods (NSFDMs). These NSFDMs are explored by many researchers to solve problems in biological sciences and other areas. Below, we mention a few of them.

Arenas et al. (2008) developed a nonstandard numerical scheme for an SIR (where S, I and R stand for susceptible, infected and removed individuals, respectively) seasonal epidemiological model for Respiratory Syncytial Virus (RSV). They compared their technique with some well-known explicit methods and carried out some simulations with data from Gambia and Finland. They showed that the forward Euler and fourth order Runge-Kutta schemes do not converge unless the step-size used in the numerical simulations for these two methods is less than a critical step-size $h_{c}=0.1$.

Some useful studies on dynamical systems are those by found in the works of Dulęba (2004), Rauh et al., (2009; 2009), as well as Zhai and Michel (2004). General two-dimensional autonomous dynamical systems and their standard numerical discretizations are considered by Dimitrov and Kojouharov (2005) who designed and analyzed nonstandard stability-preserving 
finite-difference schemes based on the explicit and implicit Euler and the second-order Runge-Kutta methods. The methods proposed in that paper can be applicable for solving arbitrary two-dimensional autonomous dynamical systems. In another work, Dimitrov and Kojouharov (2006), formulated positive and elementary stable nonstandard finite-difference methods to solve a general class of Rosenzweig-MacArthur predator-prey systems which involve a logistic intrinsic growth of the prey population. Their methods preserve the positivity of solutions and the stability of the equilibria for arbitrary step-sizes, while the approximations obtained by the other numerical methods experience difficulties in preserving either the stability or the positivity of the solutions, or both.

Gumel et al. (2005) investigated a class of NSFDMs for solving systems of differential equations arising in mathematical biology. They showed that their methods can often give numerical results that are asymptotically consistent with those of the corresponding continuous model by using a number of case studies in human epidemiology and ecology.

Some fundamental concepts and applications of nonstandard finite difference schemes for the solution of an initial value problem of ordinary differential equations are presented by Ibijola et al. (2008). They stated the reason why nonstandard methods are needed despite the fact that we have numerous standard methods available by pointing out that one of the shortcomings of standard methods is that qualitative properties of the exact solution are not usually transferred to the numerical solution.

Jódar et al. (2008) explained how to construct two competitive implicit finite difference schemes for a deterministic mathematical model associated with the evolution of influenza in human population. They obtained numerical simulations with different sets of initial conditions, parameter values, and time step-sizes.

Villanueva et al. (2008) developed (and analyzed numerically) nonstandard finite difference schemes which are free of numerical instabilities, to obtain the numerical solution of a mathematical model of infant obesity with constant population size. This model consists of a system of coupled nonlinear ordinary differential equations describing the dynamics of overweight and obese populations. The numerical results presented in that paper showed that their methods have better convergence properties when compared to the classical Euler or the fourth-order Runge-Kutta methods and the MATLAB routines in the sense that these routines give negative values for some of the state variables.

The relationship between a continuous dynamical system and numerical methods to solve it, viewed as discrete dynamical systems, is studied by Anguelov et al. (2009). In this work, the authors further categorized the term 'dynamic consistency' as the 'topological dynamic consistency' and proposed a topologically dynamically consistent nonstandard finite difference method.

Applications of these NSFDMs for singularly perturbed problems can be seen in the work of Kadalbajoo et al. (2006), Lubuma and Patidar (2006; 2007c; 2007a; 2007b), Munyakazi and Patidar (2010), Patidar and Sharma (2006a; 2006b), or Patidar (2008). However, an exhaustive account of work that use such methods is provided in the survey article by Patidar (2005).

In this paper, we consider a mathematical model of HIV infection proposed by Bacaer et al. (2008). The governing model is an initial value problem for a system of nonlinear ordinary differential equations. It describes the dynamics of HIV epidemic by partition of human population into susceptibles and infectious subpopulations, denoted respectively by $S$ and $I$. We modify this model by incorporating a function of the Hill type for the transmission rate of HIV rather than the function $e^{-\lambda H}$ used by Bacaer et al. (2008), where $\lambda$ is a parameter representing the behavioral change and $H$ denotes the HIV prevalence.

We develop some NSFDMs for numerical solution of a nonlinear system of ordinary differential equations describing the transmission dynamics of HIV. To the best of our knowledge, this is the first time that these NSFDMs are designed for this system. To keep the methods fully explicit, we will use the forward difference approximations for the first derivative terms. The nonlocal approximations will be used to tackle the nonlinear terms. In some cases, we will also make use of denominator functions which are a little more complex functions of the time step-size than the classical one. Furthermore, we will show that these NSFDMs preserve some key properties of the corresponding continuous model. It should be noted that the proposed schemes are unconditionally stable.

The rest of this paper is organized as follows. The continuous model and its stability properties are discussed in Section 2. In Section 3 we design and analyze a numerical method to solve the model proposed in Section 2. Further numerical analyses as well as some numerical simulations are presented in Section 4. A thorough discussion on the results as well as the scope for some future research is presented in Section 5

\section{Mathematical model}

In this paper, we will consider the mathematical model for HIV proposed by Bacaer et al. (2008) that describes the dynamics of HIV epidemic. The total human population is divided into susceptibles and infectious subpopulations denoted by $S$ and $I$, respectively. It is assumed that, at any time $t$, new recruits enter the susceptible class at a constant rate $B$. Upon effective contact with an infectious individual at time $t$, a susceptible individual acquires infection and moves into the class $I$ of infectious 
Table 1. Description and values of parameters used in the system (1).

\begin{tabular}{|l|c|l|}
\hline Description & Parameter & Value \\
\hline \hline Birth rate & $B$ & $200 /$ year \\
Maximum transmission rate & $d$ & $0.7 /$ year \\
Parameter representing & $\lambda_{0}$ & 5.9 \\
behavior change & & \\
Natural death rate & $\mu_{1}$ & $0.02 /$ year \\
Mortality rate of infectious & $\mu_{2}$ & $0.1 /$ year \\
individuals & $k$ & variable \\
Hill coefficient & $k$ \\
\hline
\end{tabular}

individuals. The effective contact rate at time $t$ is denoted by $f(H(t))$. It is a decreasing function of the HIV prevalence $H$ that reflects a reduction in risky behavior resulting from the awareness of individuals to a higher HIV prevalence. The death rates of susceptible and infected individuals are respectively denoted by $\mu_{1}$ and $\mu_{2}$. Using these notations and terminology, the governing model is described by the following nonlinear system of ordinary differential equations:

$$
\begin{aligned}
& \frac{\mathrm{d} S(t)}{\mathrm{d} t}=B-f(H(t)) H(t) S(t)-\mu_{1} S(t), \\
& \frac{\mathrm{d} I(t)}{\mathrm{d} t}=f(H(t)) H(t) S(t)-\mu_{2} I(t),
\end{aligned}
$$

where $H(t)$ is the prevalence of HIV given by

$$
H(t)=\frac{I(t)}{N(t)}
$$

with $N(t)=S(t)+I(t)$ as the total number of population.

The function $f(H)$ in this model is considered to be the following function of the Hill type:

$$
f(H)=\frac{d}{1+\lambda_{0} H^{k}}, \quad k \geq 1,
$$

where $d, \lambda_{0}$ and $k$ are real numbers and defined in Table 1 The descriptions of the state variables and other time-invariant parameters as well as their values (as presented by Bacaer et al. (2008)) are given in Table 1 below.

Remark 1. It should be noted that the model (1) does not distinguish the progression stages of HIV infection. The aim of this work is to focus on the impact of the response function on the dynamics of the model where estimates of the parameters are considered averages over all stages as indicated by Bacaer et al. (2008). However, regarding the stage progression property, the readers may refer to the work of Gumel et al. (2006) and the references therein.

The following results, which determine the basic qualitative features of the continuous model (1), will be useful in designing a robust nonstandard finite difference method for solving (1). These results (which are easy to prove) will further be used in measuring the competitiveness of NSFDMs.

Theorem 1. The set

$$
\mathcal{D}=\left\{(S, I) \in \mathbb{R}_{+}^{2}: S+I \leq \frac{B}{\mu_{1}}\right\}
$$

is positivity-invariant for the system (1).

The basic reproduction number of the system (1) is given by

$$
R_{0}=\frac{d}{\mu_{2}}
$$

To find the equilibria of system (1), we notice that, in terms of the prevalence of HIV at an equilibrium

$$
H^{*}=\frac{I^{*}}{S^{*}+I^{*}}
$$

the system (1) has the following characteristic equation:

$$
H^{*}\left(\mu_{2} \lambda_{0} H^{* k}+d H^{*}+\mu_{2}\left(1-R_{0}\right)\right)=0,
$$

where $H^{*}=0$ corresponds to the disease free equilibrium

$$
E_{0}^{*}=\left(\frac{B}{\mu_{1}}, 0\right)
$$

and any (as we can have more, one for each $H^{*}$ ) endemic equilibrium is given by

$$
E^{*}=\left(\frac{B\left(1-H^{*}\right)}{\mu_{1}\left(1-H^{*}\right)+\mu_{2} H^{*}}, \frac{B H^{*}}{\mu_{1}\left(1-H^{*}\right)+\mu_{2} H^{*}}\right),
$$

where $H^{*}$ is a positive solution of the equation

$$
\mu_{2} \lambda_{0} H^{* k}+d H^{*}+\mu_{2}\left(1-R_{0}\right)=0 .
$$

However, the above endemic equilibrium will be unique if $R_{0}>1$. (Note that, in this case, the function defined by Eqn. (8) is increasing, which means that there is only one endemic equilibrium point.) In summary,

- if $R_{0} \leq 1$, then only the disease free equilibrium exists;

- if $R_{0}>1$, then there exists a unique endemic equilibrium.

As a consequence of the above results, we have the following theorem.

Theorem 2. For any value of the Hill coefficient $k$, the system (1) exhibits a transcritical bifurcation. 
Now, for $k=1$, we provide the equilibria and their basic stability properties for the system (1). While the disease free equilibrium is given by (6), the unique endemic equilibrium of this system reads as

$$
E^{*}=\left(E_{1}^{*}, E_{2}^{*}\right)
$$

which exists only if $R_{0}>1$, where

$$
\begin{aligned}
E_{1}^{*} & =\frac{B\left(1+\lambda_{0}\right)}{\mu_{1}\left(1+\lambda_{0}\right)+\mu_{2}\left(R_{0}-1\right)} \\
E_{2}^{*} & =\frac{B\left(R_{0}-1\right)}{\mu_{1}\left(1+\lambda_{0}\right)+\mu_{2}\left(R_{0}-1\right)} .
\end{aligned}
$$

It can further be proved that these equilibria have the following stability properties:

Theorem 3. The disease free equilibrium of the system (1), $E_{0}^{*}$, is locally asymptotically stable if $R_{0}<1$ and unstable if $R_{0}>1$.

Theorem 4. The disease free equilibrium of the system (1), $E_{0}^{*}$, is globally asymptotically stable in $\mathcal{D}$ if $R_{0}<1$.

Theorem 5. The endemic equilibrium of the system (1), $E_{1}^{*}$, is locally asymptotically stable if $R_{0}>1$.

Regarding the cases when $k>1$, it can be seen from (8) that the analytical evaluation of equilibria becomes algebraically cumbersome. We therefore evaluate them numerically, and we will also investigate some of the properties that they possess. This will be done in Section 4.1

\section{Construction and analysis of the NSFDM}

In this section, we design a nonstandard finite difference method that satisfies the positivity of the state variables involved in the system. It is important that a numerical method preserves this property when used to solve differential models arising in population biology because these state variables represent subpopulations which never take negative values.

To begin with, the time domain $[0, T]$ is partitioned through the discrete time levels $t_{n}=n \ell$, where $\ell>0$ is the time step-size.

To construct the NSFDM, we discretized the system (1) based on the approximation of the temporal derivatives by a generalized first order forward difference method as follows. by

For $S(t) \in \mathbb{C}^{1}(\mathbb{R})$, the discrete derivative is defined

$$
\frac{\mathrm{d} S(t)}{\mathrm{d} t}=\frac{S(t+\ell)-S(t)}{\psi(\ell)}+\mathcal{O}(\psi(\ell)) \quad \text { as } \ell \rightarrow 0,
$$

where $\psi(\ell)$ is a denominator function (Mickens and Smith, 1990; 2007) which is a real-valued function and satisfies

$$
\psi(\ell)=\ell+\mathcal{O}\left(\ell^{2}\right) \text { for all } \ell>0 .
$$

The discrete derivative for $I(t)$ is obtained analogously whereas the non-derivative terms are approximated locally, i.e., at the base time level.

Denoting the approximations of $S(n h)$ and $I(n h)$ by $S^{n}$ and $I^{n}$, respectively, where $n=0,1,2, \ldots$, the NSFDM reads

$$
\begin{aligned}
& \frac{S^{n+1}-S^{n}}{\psi(\ell)}=B-\mu_{1} S^{n+1}-f\left(H^{n}\right) H^{n} S^{n+1}, \\
& \frac{I^{n+1}-I^{n}}{\psi(\ell)}=f\left(H^{n}\right) H^{n} S^{n+1}-\mu_{2} I^{n+1},
\end{aligned}
$$

where discretizations for $H$ and $f(H)$ are given by

$$
H^{n}=\frac{I^{n}}{S^{n}+I^{n}}
$$

and

$$
f\left(H^{n}\right)=\frac{d}{1+\lambda_{0}\left(H^{n}\right)^{k}},
$$

respectively.

Remark 2. It is to be noted that, besides the use of a non-classical denominator function, we have also used some non-local discretizations. As mentioned in the literature (see, e.g., Mickens and Ramadhani, 1994; Patidar, 2005) a finite difference method is termed a nonstandard finite difference method if either we use a denominator function or a non-local approximation. In view of this, when $\psi(\ell)=\ell$, the above method will be referred to as "NSFDM-I". However, if the denominator function $\psi(\ell)$ is different from $\ell$, the method will be referred to as "NSFDM-II". In this work, this function is considered to be $\left(e^{\mu_{2} \ell}-1\right) / \mu_{1}, \mu_{2}>\mu_{1}$.

Simplifying (12), we obtain

$$
\begin{aligned}
S^{n+1} & =\frac{S^{n}+\psi(\ell) B}{1+\psi(\ell)\left\{f\left(H^{n}\right) H^{n}+\mu_{1}\right\}}, \\
I^{n+1} & =\frac{I^{n}+\psi(\ell) f\left(H^{n}\right) H^{n} S^{n+1}}{1+\mu_{2} \psi(\ell)} .
\end{aligned}
$$

The positivity of the solution reflects from the above method (15), because if the initial values $S(0)$ and $I(0)$ are non-negative then the right hand side of (15) admits no negative terms for any of $n=0,1,2,3, \ldots$.

In the following section we determine the stability properties of the system (12), and we verify that

(i) the continuous and the discrete models have the same equilibria, and

(ii) both models possess similar qualitative features near these equilibria. 
3.1. Fixed points and stability analysis. We study in this section the stability and convergence properties of the fixed points of the proposed NSFDM numerical method (12).

We begin by noting that the fixed points $(\hat{S}, \hat{I})$ of the system (12) can be found by solving

$$
\begin{aligned}
& F(\hat{S}, \hat{I})=\hat{S} \\
& G(\hat{S}, \hat{I})=\hat{I}
\end{aligned}
$$

where $F(\hat{S}, \hat{I})$ and $G(\hat{S}, \hat{I})$ can be obtained by considering the right hand sides in (15), i.e.,

$$
\begin{aligned}
& F(\hat{S}, \hat{I})=\frac{\hat{S}+\psi(\ell) B}{1+\psi(\ell)\left\{f(\hat{H}) \hat{H}+\mu_{1}\right\}}, \\
& G(\hat{S}, \hat{I})=\frac{\hat{I}+\psi(\ell) f(\hat{H}) \hat{H} \hat{S}}{1+\mu_{2} \psi(\ell)},
\end{aligned}
$$

where

$$
\hat{H}=\frac{\hat{I}}{\hat{S}+\hat{I}} .
$$

Solving (16), we obtain the following equation for $\hat{H}$ :

$$
\hat{H}\left(\mu_{2} \lambda_{0} \hat{H}^{k}+d \hat{H}+\mu_{2}\left(1-R_{0}\right)\right)=0 .
$$

In the above equation, $\hat{H}=0$ corresponds to the disease free equilibrium

$$
\hat{E}_{0}=\left(\frac{B}{\mu_{1}}, 0\right),
$$

whereas any endemic equilibrium is given by

$$
\hat{E}=\left(\frac{B(1-\hat{H})}{\mu_{1}(1-\hat{H})+\mu_{2} \hat{H}}, \frac{B \hat{H}}{\mu_{1}(1-\hat{H})+\mu_{2} \hat{H}}\right),
$$

in which $\hat{H}$ corresponds to the positive solutions of the characteristic equation

$$
\mu_{2} \lambda_{0} \hat{H}^{k}+d \hat{H}+\mu_{2}\left(1-R_{0}\right)=0 .
$$

The form of the above equation is similar to the characteristic equation for the continuous systems (1) given by (8). Therefore, both the systems (1) and (12) have the same characteristic equation and expressions of equilibria. Hence, we have the following result.

Remark 3. For any $k$, the continuous system (1) and the discrete system (12) have the same equilibria. Furthermore, when $k=1$, then, in addition to the above disease free fixed point, the system (12) has the following endemic fixed point:

$$
\hat{E}=\left(\frac{B\left(1+\lambda_{0}\right)}{\hat{E}_{1}^{d}}, \frac{B\left(R_{0}-1\right)}{\hat{E}_{1}^{d}}\right),
$$

which exists only if $R_{0}>1$, where

$$
\hat{E}_{1}^{d}=\mu_{1}\left(1+\lambda_{0}\right)+\mu_{2}\left(R_{0}-1\right) .
$$

The next theorems give us the stability properties only when $k=1$. However, it is difficult to find the endemic fixed points for the system (12) in closed form when $k \geq 2$, and we will be investigating them numerically. This will be shown in Section 4.2. Moreover, we will show that both systems (discrete as well as continuous) behave similarly near their equilibria.

Theorem 6. Let $\psi(\ell)$ be a real-valued function such that $\psi(\ell)=\ell+O\left(\ell^{2}\right)$. If $R_{0}<1$, then the system (12) is unconditionally (i.e., regardless of the step-size $\ell$ ) locally asymptotically stable at the disease free equilibrium, $\hat{E}_{0}=\left(B / \mu_{1}, 0\right)$, and unstable otherwise.

Proof. The Jacobian matrix of the system (12) evaluated at the disease free equilibrium, $\hat{E}_{0}$, is

$$
J\left(\hat{E}_{0}\right)=\left(\begin{array}{cc}
\frac{1}{1+\psi(\ell) \mu_{1}} & -\frac{\psi(\ell) d}{1+\psi(\ell) \mu_{1}} \\
0 & \frac{1+\psi(\ell) d}{1+\psi(\ell) \mu_{2}}
\end{array}\right) .
$$

Being a triangular matrix, its eigenvalues are the entries along the main diagonal, i.e.,

$$
\lambda_{1}=\frac{1}{1+\psi(\ell) \mu_{1}}, \quad \lambda_{2}=\frac{1+\psi(\ell) d}{1+\psi(\ell) \mu_{2}} .
$$

It should be noted that the inequality $\left|\lambda_{1}\right|<1$ always holds since $R_{0}<1$ for the disease free equilibrium. Hence, the spectral radius is strictly less than unity in magnitude if $R_{0}<1$ for all $\ell$, and then, using Theorem 2.10 of Allen (2007), the required result is obtained.

Theorem 7. The endemic equilibrium of the system (12), $\hat{E}$, is unconditionally locally asymptotically stable if $R_{0}>1$.

Proof. The Jacobian matrix of the system 12 evaluated at the endemic equilibrium (23) is

$$
J(\hat{E})=\left(\begin{array}{cc}
\frac{R_{0}\left(1+\lambda_{0}\right)+\psi(\ell) \mu_{2}\left(R_{0}-1\right)}{J_{1}^{d}} & -\frac{\mu_{2}\left(1+\lambda_{0}\right) \psi(\ell)}{J_{1}^{d}} \\
\frac{\mu_{2}\left(R_{0}-1\right)^{2} \psi(\ell)}{R_{0}\left(1+\psi(\ell) \mu_{2}\right)\left(1+\lambda_{0}\right)} & \left.\frac{R_{0}+\psi(\ell) \mu_{2}}{R_{0}\left(1+\psi(\ell) \mu_{2}\right)}\right)
\end{array}\right),
$$

where

$$
J_{1}^{d}=R_{0}\left(\left(1+\psi(\ell) \mu_{1}\right)\left(1+\lambda_{0}\right)+\psi(\ell) \mu_{2}\left(R_{0}-1\right)\right),
$$

which can be written in the following form:

$$
J(\hat{E})=\left(\begin{array}{cc}
b_{1} & -b_{2} \\
b_{3} & b_{4}
\end{array}\right),
$$


where

$$
\begin{aligned}
& b_{1}=\frac{a_{1}+a_{2}}{a_{1} a_{3}+R_{0} a_{2}}, \\
& b_{2}=\frac{a_{4}}{a_{1} a_{3}+R_{0} a_{2}}, \\
& b_{3}=\frac{a_{2}\left(R_{0}-1\right)}{a_{5} a_{7}}, \\
& b_{4}=\frac{a_{6}}{a_{7}},
\end{aligned}
$$

with

$$
\begin{aligned}
& a_{1}=R_{0}\left(1+\lambda_{0}\right), \\
& a_{2}=\psi(\ell) \mu_{2}\left(R_{0}-1\right), \\
& a_{3}=1+\psi(\ell) \mu_{1}, \\
& a_{4}=\mu_{2}\left(1+\lambda_{0}\right) \psi(\ell), \\
& a_{5}=1+\lambda_{0}, \\
& a_{6}=R_{0}+\psi(\ell) \mu_{2}, \\
& a_{7}=R_{0}\left(1+\psi(\ell) \mu_{2}\right) .
\end{aligned}
$$

Since we have $R_{0}>1$, it follows that $a_{i}>0,1 \leq i \leq 7$, $0<b_{1}, b_{4}<1$ and $b_{2}, b_{3}>0$.

The characteristic equation associated with the above matrix is given by

$$
g(\lambda)=\lambda^{2}-A_{1} \lambda+A_{2}=0
$$

where

$$
A_{1}=b_{1}+b_{4}>0 \text { and } A_{2}=b_{1} b_{4}+b_{2} b_{3}>0 .
$$

Lemma 1. (Brauer and Castillo-Chavez, 2001) The roots of Eqn. (24) satisfy $\left|\lambda_{i}\right|<1, i=1$, 2, if and only if the following conditions are satisfied:

1. $g(1)=1-A_{1}+A_{2}>0$,

2. $g(-1)=1+A_{1}+A_{2}>0$,

3. $g(0)=A_{2}<1$.

From Eqn. (24), we have

$$
\begin{aligned}
g(1) & =1-A_{1}+A_{2}, \\
& =\left(1-b_{1}\right)\left(1-b_{4}\right)+b_{2} b_{3}, \\
& >0,
\end{aligned}
$$

as both $b_{1}$ and $b_{4}$ are less than unity. Also

$$
\begin{aligned}
g(-1) & =1+A_{1}+A_{2}, \\
& >0,
\end{aligned}
$$

since both $A_{1}$ and $A_{2}$ are greater than zero. Moreover, we have

$$
\begin{aligned}
g(0) & =A_{2}, \\
& =\frac{\nu_{1}+\nu_{2}}{\nu_{1}+R_{0} \nu_{2}+\nu_{3}}<1,
\end{aligned}
$$

since $R_{0}>1$, where

$$
\begin{aligned}
& \nu_{1}=R_{0}\left(1+\lambda_{0}\right)>0, \\
& \nu_{2}=\ell \mu_{2}\left(R_{0}+\lambda_{0}\right)+\ell^{2} \mu_{2}^{2}\left(R_{0}-1\right)>0, \\
& \nu_{3}=\ell \mu_{1} R_{0}\left(1+\lambda_{0}\right)\left(1+\ell \mu_{2}\right)>0 .
\end{aligned}
$$

From (25)-(27), the conditions of Lemma 1 hold. Therefore, the eigenvalues of the associated Jacobian matrix in this case are strictly less than unity in modulus when $R_{0}>1$ for all step-sizes $\ell$. Hence, the numerical method (12) is unconditionally stable at its endemic equilibrium $\hat{E}$.

Remark 4. From the results in this section, we can conclude that both models (the continuous one (1) as well as the discrete one (12) have the same equilibria, and their behavior is qualitatively similar near these equilibria. Therefore, the nonstandard finite difference method (12) is elementary stable.

\section{Numerical results and simulations}

We present some numerical simulations using the proposed NSFDMs in this section. The numerical results that we obtain support our theoretical results. The methods are also tested for convergence. We numerically show that both of these methods (NSFDM-I and NSFDM-II) are elementary stable when the Hill coefficient $k \geq$ 2. A number of different numerical simulations are carried out and comparisons are made with other well-known numerical methods for various time step-sizes $\ell$. Parameter values used in the simulations are presented in Table 1 Some of these parameters are varied to test the robustness of the methods. As mentioned in Section 2. we attempt to investigate the endemic equilibria and their stability for both continuous and discrete models numerically as shown below in Tables 24, Parameters used for this part of the simulations are taken from Table 1 which gives $R_{0}>1$.

4.1. Numerical stability analysis of the endemic equilibria. In this section, we tabulate the equilibria and their respective eigenvalues associated with the Jacobian matrices for the continuous system (1) for different values of Hill coefficient $k$. It should be noted that, when solving the system (1) for its equilibria when $k=2,3, \ldots, 10$, it always has the disease free equilibrium $E_{0}^{*}=(10000,0)$ and $k$ endemic equilibria (for the set of parameter values presented in Table 1, which give $\left.\left(R_{0}>1\right)\right)$, but there is only one endemic equilibrium point for each value of $k$. It is clear from the above tabular results that, the eigenvalues in each case of $k=2,3, \ldots, 10$ are negative. We therefore have the following remark.

Remark 5. For $k=2,3,4, \ldots, 10$, the system (1) has a disease free equilibrium when $R_{0}<1$ and it possesses a 
Table 2. Endemic equilibria and their eigenvalues for the system (1) when $k \geq 2$.

\begin{tabular}{|c|c|c|c|c|}
\hline$k$ & $S^{*}$ & $I^{*}$ & $\lambda_{1}$ & $\lambda_{2}$ \\
\hline \hline 2 & 1280 & 1744 & -0.1998 & -0.0890 \\
3 & 1019 & 1796 & -0.2903 & -0.0876 \\
4 & 866 & 1827 & -0.3652 & -0.0881 \\
5 & 763 & 1847 & -0.4288 & -0.0889 \\
6 & 689 & 1862 & -0.4832 & -0.0897 \\
7 & 633 & 1873 & -0.5296 & -0.0904 \\
8 & 589 & 1882 & -0.5690 & -0.0910 \\
9 & 553 & 1889 & -0.6022 & -0.0916 \\
10 & 523 & 1895 & -0.6298 & -0.0922 \\
\hline
\end{tabular}

number of endemic equilibria as presented above in Table 2 when $R_{0}>1$. Each of these endemic equilibria is locally asymptotically stable if $R_{0}>1$.

4.2. Numerical stability analysis of the fixed points. The spectral radii of the Jacobian matrices corresponding to the fixed points of the numerical method for different values of Hill coefficient $k$ and the time step-size $\ell$ are tabulated in this section. We recall from Remark 3 that the equilibria of both systems (1) and (12) remain the same for any value of $k$.

It can be seen from the two tables above that all the spectral radii are less than one in magnitude irrespective of the time step-size used in the simulations. Hence, each of these fixed points is locally asymptotically stable if $R_{0}>1$ for $k=2,3, \ldots, 10$. Thus, we have the following remark.

Remark 6. For $k=2,3, \ldots, 10$, each fixed point of system 12 is locally asymptotically stable if $R_{0}>$ 1. Moreover, the system is unconditionally elementary stable.

4.3. Numerical simulations for the disease free equilibrium. The Disease Free Equilibrium (DFE) is calculated using the proposed NSFDMs along with other numerical methods conventionally used. A thorough comparison of these methods is presented for many different scenarios. The maximum transmission rate, $d$, is very important from the biological point of view and hence its value will be varied in a certain range while keeping $R_{0}<1$ (as needed for the DFE).

In Section 2, we showed that system (1) has an asymptotically stable disease free equilibrium if $R_{0}<1$. The numerical value of this DFE is given by $E_{0}^{*}=$ $(10000,0)$. In order to check whether these numerical methods converge to the theoretical value of the DFE, we require a tolerance value. To this end, for the susceptible we consider their value as $1 \%$ of the susceptible population whereas we consider 20 individuals as the tolerance value for the infectious population. Although all the numerical methods converge to the disease free equilibrium, $E_{0}^{*}$, for any time step-size used when $d=$ 0.07, we can see from Fig. 1 that only NSFDM-II achieves much better convergence. In Table 5] as well as in Fig. 2, it is shown when $d=0.09$ only NSFDM-II converges to the correct disease free equilibrium, $E_{0}^{*}$, for different values of the step-size.

To see the robustness of the NSFDMs with respect to the initial conditions, the results are presented in Table 6 . In this table we also put the results obtained by the fourth order Runge-Kutta method and the MATLAB solver ode45. It can be seen that NSFDM-II converges for all initial conditions whereas the others do not. This also can be seen in Fig. 3

As far as the positivity of the solutions obtained by these methods is concerned, we note that the Euler method does not preserve this property although it converges for a wide range of the step-sizes and initial conditions (see, Fig. (4). However, NSFDMs always preserve this property.

4.4. Numerical simulations for the endemic equilibria. In this section, we study the convergence behavior of numerical methods to endemic equilibria. We provide the results for various values of the Hill coefficient $k$.

4.4.1. Case I: Hill coefficient $\boldsymbol{k}=\mathbf{1}$. When $k=1$, the unique endemic equilibrium, $E^{*}$, of the system (1) is locally asymptotically stable if $d>\mu_{2}\left(R_{0}>1\right)$. In this section, the tolerance values are $1 \%$ and $10 \%$ for $S^{*}$ and $I^{*}$, respectively.

It can be seen from Figs. 5 and 6 that all numerical methods converge almost to the endemic equilibrium $E^{*}$ when $d>\mu_{2}$. However, NSFDM-II converges more accurately.

Furthermore, all the numerical methods converge to the correct endemic equilibrium for any initial conditions used. However, when $d$ is close to $\mu_{2}$ (which means that $R_{0}$ is close to 1 ), only NSFDM-II could achieve convergence for a wide range of the initial conditions. This is shown in Table 7 as well as in Fig. 7 Again as in the case of the DFE, the preservation of the positivity of the solutions is observed only for NSFDM-I. The Euler method failed to do so although it converges asymptotically to the correct endemic equilibrium. This is depicted in Fig. 8, below.

4.4.2. Case II: Hill coefficient $k>1$. In this section, simulation results are presented for different numerical methods for a range of $k>1$. As in the previous case, the tolerance value for $S^{*}$ is taken as $1 \%$. However, for $k>1$, there will be sufficient fluctuations in the dynamics of the infectious population and therefore we would not take the tolerance as $10 \%$ in this case, and hence the $1 \%$ tolerance would suffice for $I^{*}$. It can be seen that all the methods in 
Table 3. Spectral radii of the Jacobian matrices corresponding to the fixed points of NSFDM-I for $k \geq 2$.

\begin{tabular}{|c|c|c|c|c|c|c|}
\hline$k$ & $\ell=0.01$ & $\ell=0.5$ & $\ell=1$ & $\ell=10$ & $\ell=20$ & $\ell=100$ \\
\hline \hline 2 & 0.998864 & 0.945832 & 0.896589 & 0.431238 & 0.241651 & 0.034112 \\
3 & 0.998706 & 0.938325 & 0.882257 & 0.352413 & 0.136550 & 0.177431 \\
4 & 0.998608 & 0.933664 & 0.873359 & 0.303474 & 0.071299 & 0.266411 \\
5 & 0.998546 & 0.930702 & 0.867703 & 0.272367 & 0.029823 & 0.322969 \\
6 & 0.998508 & 0.928864 & 0.864195 & 0.253071 & 0.004094 & 0.358054 \\
7 & 0.998486 & 0.927820 & 0.862201 & 0.242107 & 0.010524 & 0.377988 \\
8 & 0.998476 & 0.927359 & 0.861322 & 0.237270 & 0.016973 & 0.386782 \\
9 & 0.998476 & 0.927340 & 0.861285 & 0.237065 & 0.017247 & 0.387155 \\
10 & 0.998482 & 0.927659 & 0.861895 & 0.240424 & 0.012768 & 0.381048 \\
\hline
\end{tabular}

Table 4. Spectral radii of the Jacobian matrices corresponding to the fixed points of NSFDM-II for $k \geq 2$.

\begin{tabular}{|c|c|c|c|c|c|c|}
\hline$k$ & $\ell=0.01$ & $\ell=0.5$ & $\ell=1$ & $\ell=10$ & $\ell=20$ & $\ell=100$ \\
\hline \hline 2 & 0.994338 & 0.767892 & 0.607976 & 0.018925 & 0.102996 & 0.137513 \\
3 & 0.993553 & 0.735724 & 0.553645 & 0.160140 & 0.255862 & 0.295163 \\
4 & 0.993066 & 0.715752 & 0.519913 & 0.247812 & 0.350768 & 0.393039 \\
5 & 0.992756 & 0.703058 & 0.498472 & 0.303540 & 0.411093 & 0.455252 \\
6 & 0.992564 & 0.695183 & 0.485172 & 0.338109 & 0.448515 & 0.493845 \\
7 & 0.992455 & 0.690708 & 0.477615 & 0.357751 & 0.469777 & 0.515773 \\
8 & 0.992407 & 0.688735 & 0.474282 & 0.366415 & 0.479157 & 0.525446 \\
9 & 0.992405 & 0.688651 & 0.474140 & 0.366783 & 0.479555 & 0.525856 \\
10 & 0.992438 & 0.690022 & 0.476455 & 0.360766 & 0.473041 & 0.519139 \\
\hline
\end{tabular}

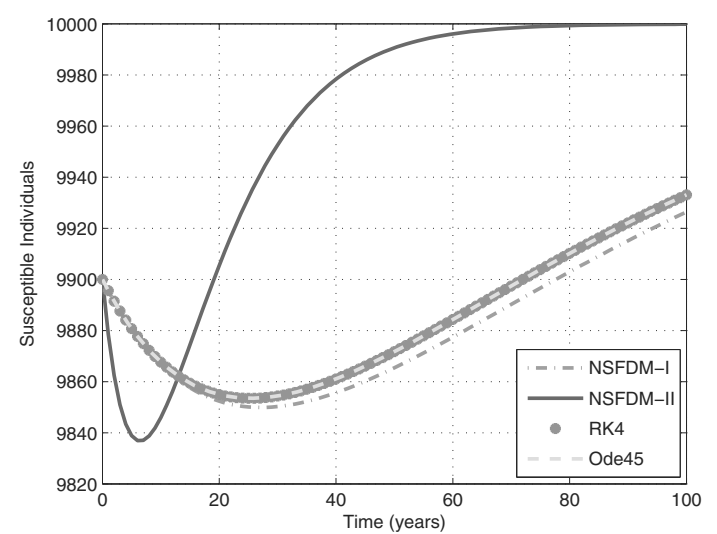

(a)

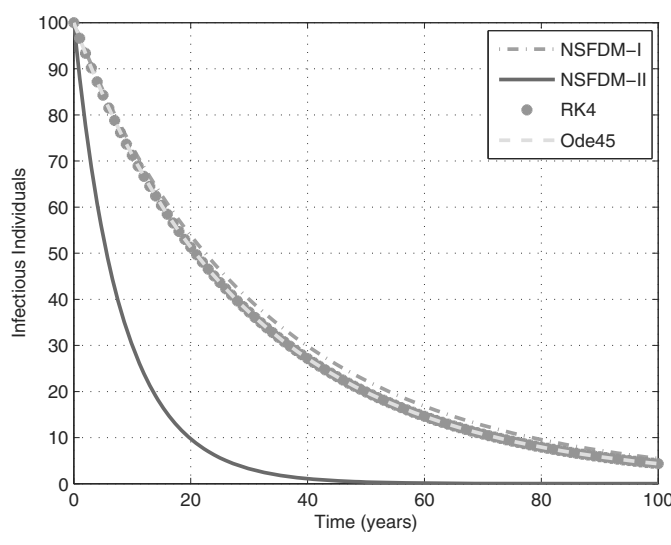

(b)

Fig. 1. Profiles of solutions (susceptibles $(S(t))$ (a) and infectious individuals $(I(t)$ ), (b)) obtained by using different numerical methods when $d=0.07$, with initial conditions $(S(0), I(0))=(9900,100) \cdot \ell=1$.

Table 5. Results obtained by different numerical methods when $d=0.09$ with initial conditions $(S(0), I(0))=(9900,100)$. The disease free equilibrium is given by $\left(S^{*}, I^{*}\right)=(10000,0)$.

\begin{tabular}{|l|c|c|c|c|}
\hline$\ell$ & ode45 & RK4 & NSFDM-I & NSFDM-II \\
\hline \hline 0.01 & Diverge & Diverge & Diverge & Converge \\
0.1 & Diverge & Diverge & Diverge & Converge \\
0.5 & Diverge & Diverge & Diverge & Converge \\
1 & Diverge & Diverge & Diverge & Converge \\
4 & Diverge & Diverge & Diverge & Converge \\
6 & Diverge & Diverge & Diverge & Converge \\
\hline
\end{tabular}



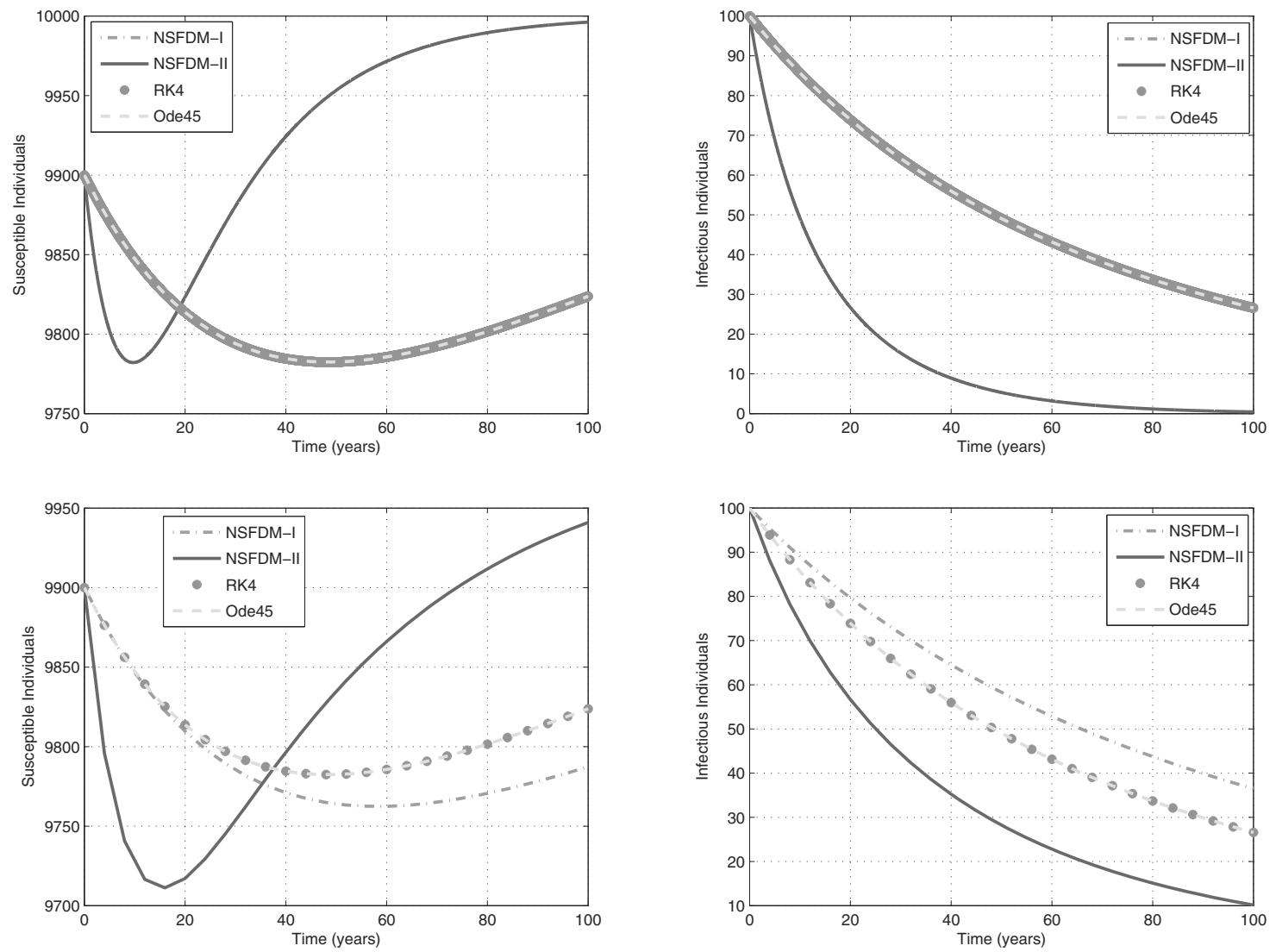

(a)

(b)

Fig. 2. Profiles of solutions (susceptibles $(S(t))$ (a) and infectious individuals $(I(t))$ (b)) obtained by using different numerical methods when $d=0.09$, with initial conditions $(S(0), I(0))=(9900,100) \cdot \ell=0.01$ (upper plots), $\ell=4$ (lower plots).

Table 6. Results obtained by different numerical methods for different initial conditions when $d=0.07$ and $\ell=1$. The disease free equilibrium is given by $\left(S^{*}, I^{*}\right)=(10000,0)$.

\begin{tabular}{|l|l|l|l|l|l|}
\hline$S(0)$ & $I(0)$ & ode45 & RK4 & NSFDM-I & NSFDM-II \\
\hline \hline 9950 & 50 & Converge & Converge & Converge & Converge \\
9900 & 100 & Converge & Converge & Converge & Converge \\
9850 & 150 & Converge & Converge & Diverge & Converge \\
9800 & 200 & Diverge & Diverge & Diverge & Converge \\
7000 & 3000 & Diverge & Diverge & Diverge & Converge \\
2000 & 8000 & Diverge & Diverge & Diverge & Converge \\
\hline
\end{tabular}

Table 7. Results obtained by different numerical methods for different initial conditions when $d=0.11$ and $\ell=1$. The disease free equilibrium is given by $\left(S^{*}, I^{*}\right)=(9324,135)$.

\begin{tabular}{|l|l|l|l|l|l|}
\hline$S(0)$ & $I(0)$ & ode45 & RK4 & NSFDM-I & NSFDM-II \\
\hline \hline 9318 & 141 & Converge & Converge & Converge & Converge \\
9314 & 145 & Converge & Converge & Converge & Converge \\
9267 & 192 & Diverge & Diverge & Diverge & Converge \\
8275 & 1184 & Diverge & Diverge & Diverge & Converge \\
6280 & 3179 & Diverge & Diverge & Diverge & Converge \\
3324 & 6135 & Diverge & Diverge & Diverge & Converge \\
\hline
\end{tabular}



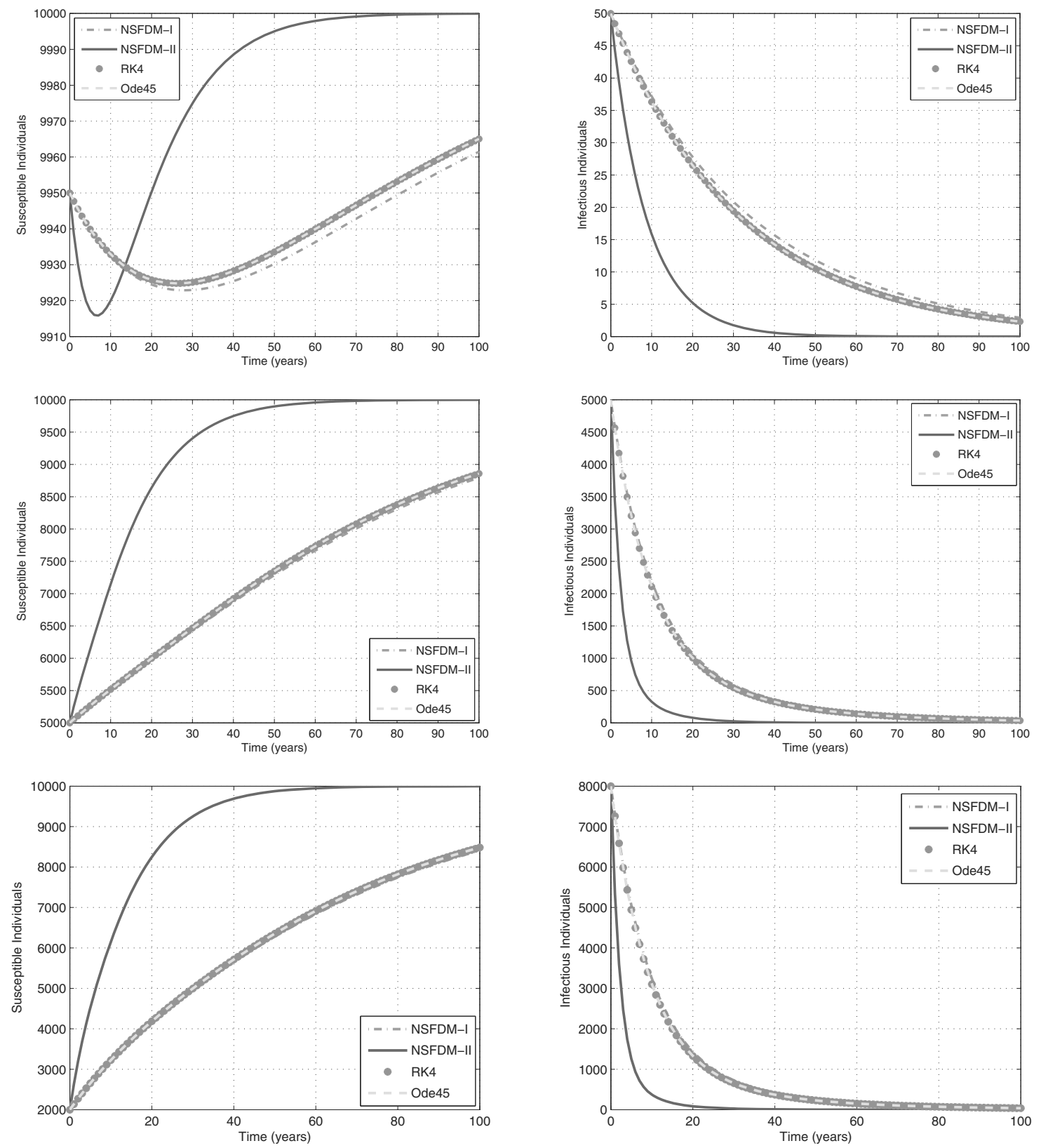

(a)

(b)

Fig. 3. Profiles of solutions (susceptibles $(S(t))$ (a) and infectious individuals $(I(t))$ (b)) obtained by using different numerical methods when $d=0.07$ and $\ell=1 .(S(0), I(0))=(9950,50)$ (upper plots), $(S(0), I(0))=(5000,5000)$ (middle plots), $(S(0), I(0))=(2000,8000)$ (lower plots).

the tables converge for small step-sizes. However, when the step-sizes are larger, then only NSFDMs converge to the correct endemic equilibrium. This is shown in Tables 8, 10 Furthermore, Figs. 9-11 show how the different numerical methods converge to the equilibrium in each case when the step-size is 1 . While all these methods converge, we see that convergence is oscillatory in the case of ode45 (see Fig. 11). NSFDM-II also oscillates in the transient face but converges much before the other methods. However, NSFDM-I has the best performance.

\section{Discussion and concluding remarks}

In this paper, an unconditionally stable nonstandard finite difference method is proposed for solving a mathematical 


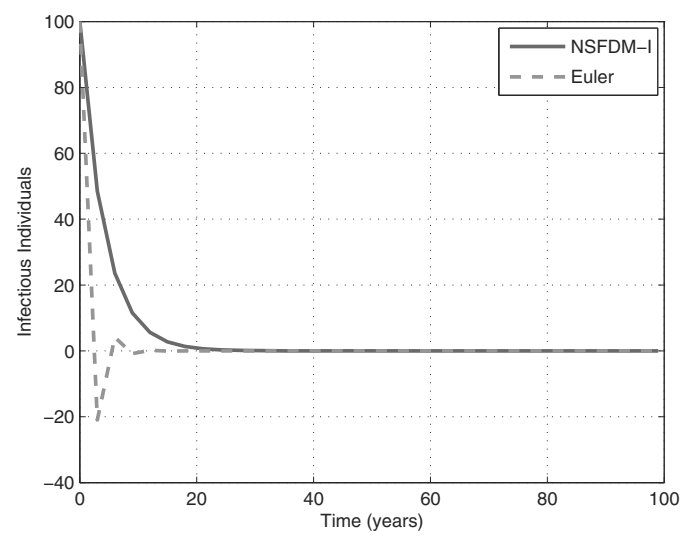

(a)

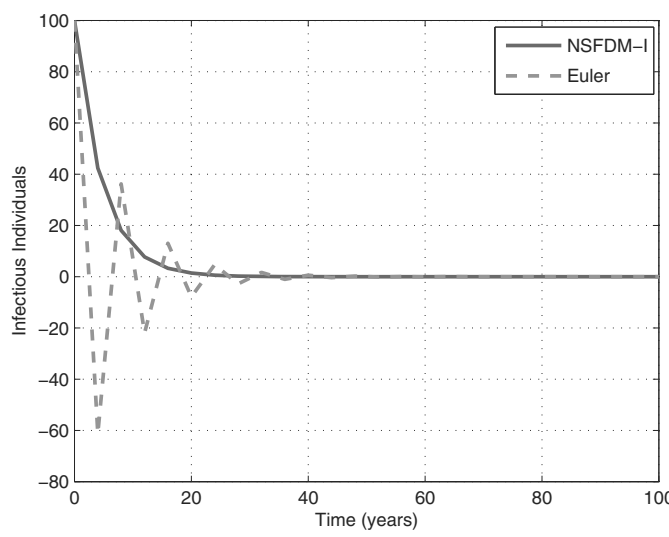

(b)

Fig. 4. Profiles of solutions for infectious individuals $(I(t))$ obtained by NSFDM-I and the Euler method when $d=0.05, \mu_{2}=0.45$, with initial conditions $(S(0), I(0))=(9900,100) \cdot \ell=3$ (a), $\ell=4$ (b).
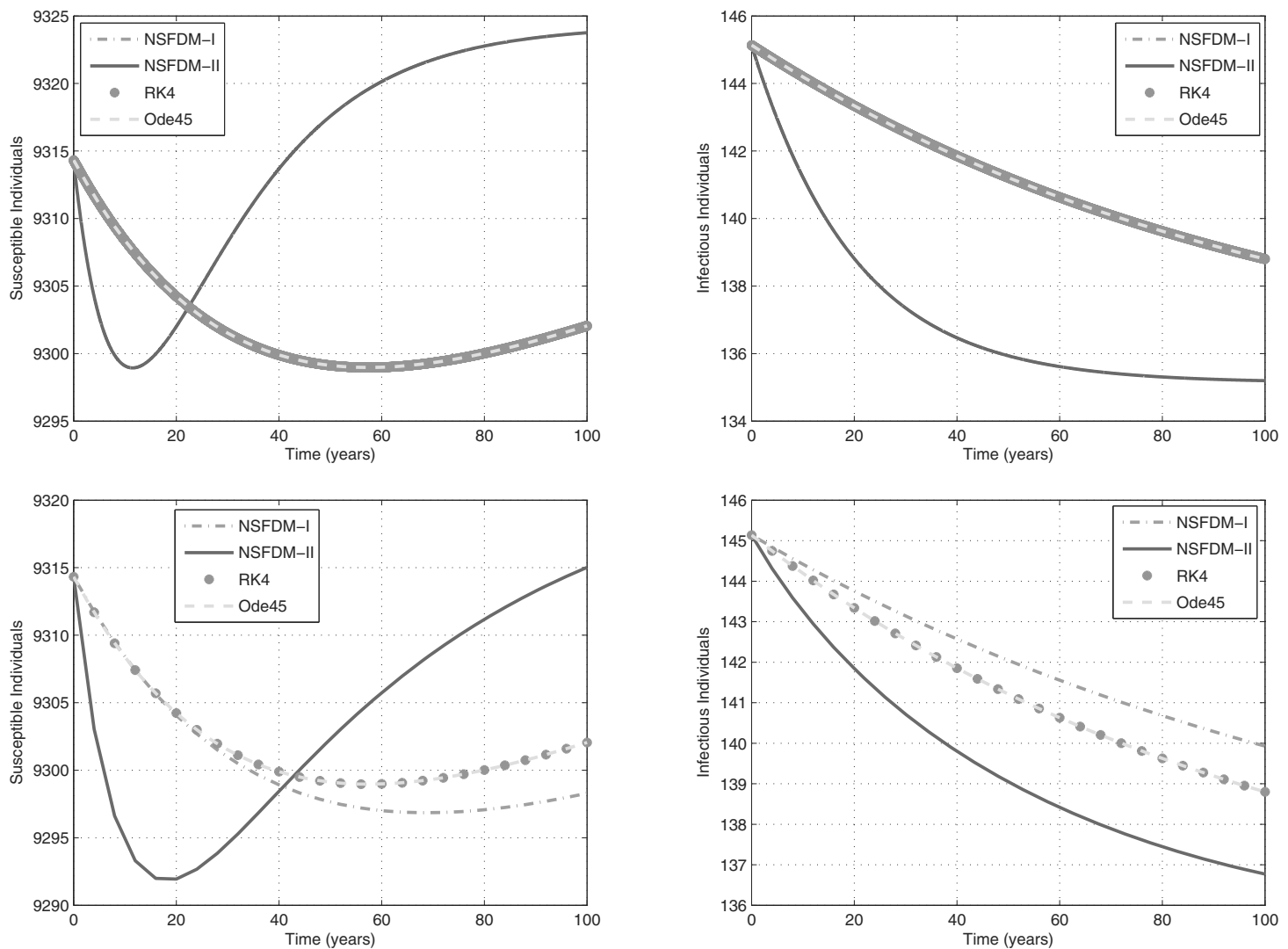

Fig. 5. Profiles of solutions (susceptibles $(S(t))$ and infectious individuals $(I(t))$ ) obtained by using different numerical methods when $d=0.11$, with initial conditions $S(0), I(0))=(9314,145) . \ell=0.01$ (upper plots), $\ell=4$ (lower plots).

model of HIV represented by a nonlinear system of ordinary differential equations. The proposed method is very competitive. It is qualitatively stable, that is, it produces results which are dynamically consistent with those of the continuous system.
Numerical results presented in Section 4 confirm further applicability of the proposed NSFDMs for biological systems. These methods preserve the positivity of solutions and the stability properties of the equilibria for arbitrary step-sizes, while the solutions obtained 

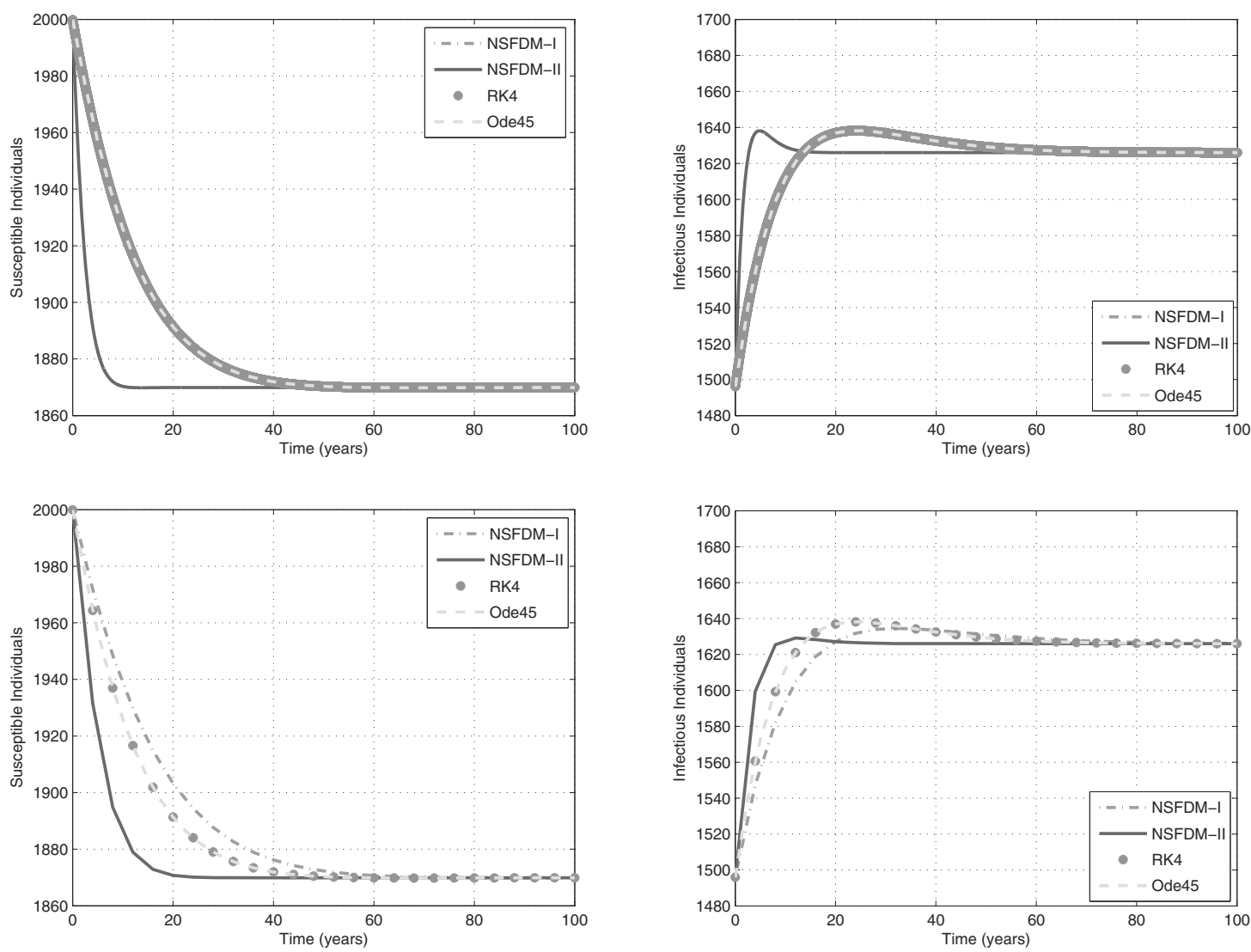

Fig. 6. Profiles of solutions (susceptibles $(S(t))$ and infectious individuals $(I(t))$ ) obtained by using different numerical methods when $d=0.7$, with initial conditions $S(0), I(0))=(2000,1496) . \ell=0.01$ (upper plots), $\ell=4$ (lower plots).

Table 8. Results obtained by different numerical methods for $k=2$ with initial conditions $(S(0), I(0))=(1324,1700)$ and different step-sizes. In this case, the endemic equilibrium is given by $\left(S^{*}, I^{*}\right)=(1280,1744)$.

\begin{tabular}{|l|l|l|l|l|}
\hline$\ell$ & ode45 & RK4 & NSFDM-I & NSFDM-II \\
\hline \hline 0.01 & Converge & Converge & Converge & Converge \\
1 & Converge & Converge & Converge & Converge \\
4 & Converge & Converge & Converge & Converge \\
8 & Converge & Converge & Converge & Converge \\
12 & Failed & Converge & Converge & Converge \\
15 & Failed & Diverge & Converge & Converge \\
20 & Failed & Diverge & Converge & Converge \\
\hline
\end{tabular}

Table 9. Results obtained by different numerical methods for $k=5$ with initial conditions $(S(0), I(0))=(810,1800)$ and different step-sizes. In this case, the endemic equilibrium is given by $\left(S^{*}, I^{*}\right)=(763,1847)$.

\begin{tabular}{|l|l|l|l|l|}
\hline$\ell$ & ode45 & RK4 & NSFDM-I & NSFDM-II \\
\hline \hline 0.01 & Converge & Converge & Converge & Converge \\
1 & Converge & Converge & Converge & Converge \\
3 & Converge & Converge & Converge & Converge \\
4 & Failed & Converge & Converge & Converge \\
6 & Failed & Converge & Converge & Converge \\
10 & Failed & Diverge & Converge & Converge \\
15 & Failed & Diverge & Converge & Converge \\
20 & Failed & Diverge & Converge & Converge \\
\hline
\end{tabular}



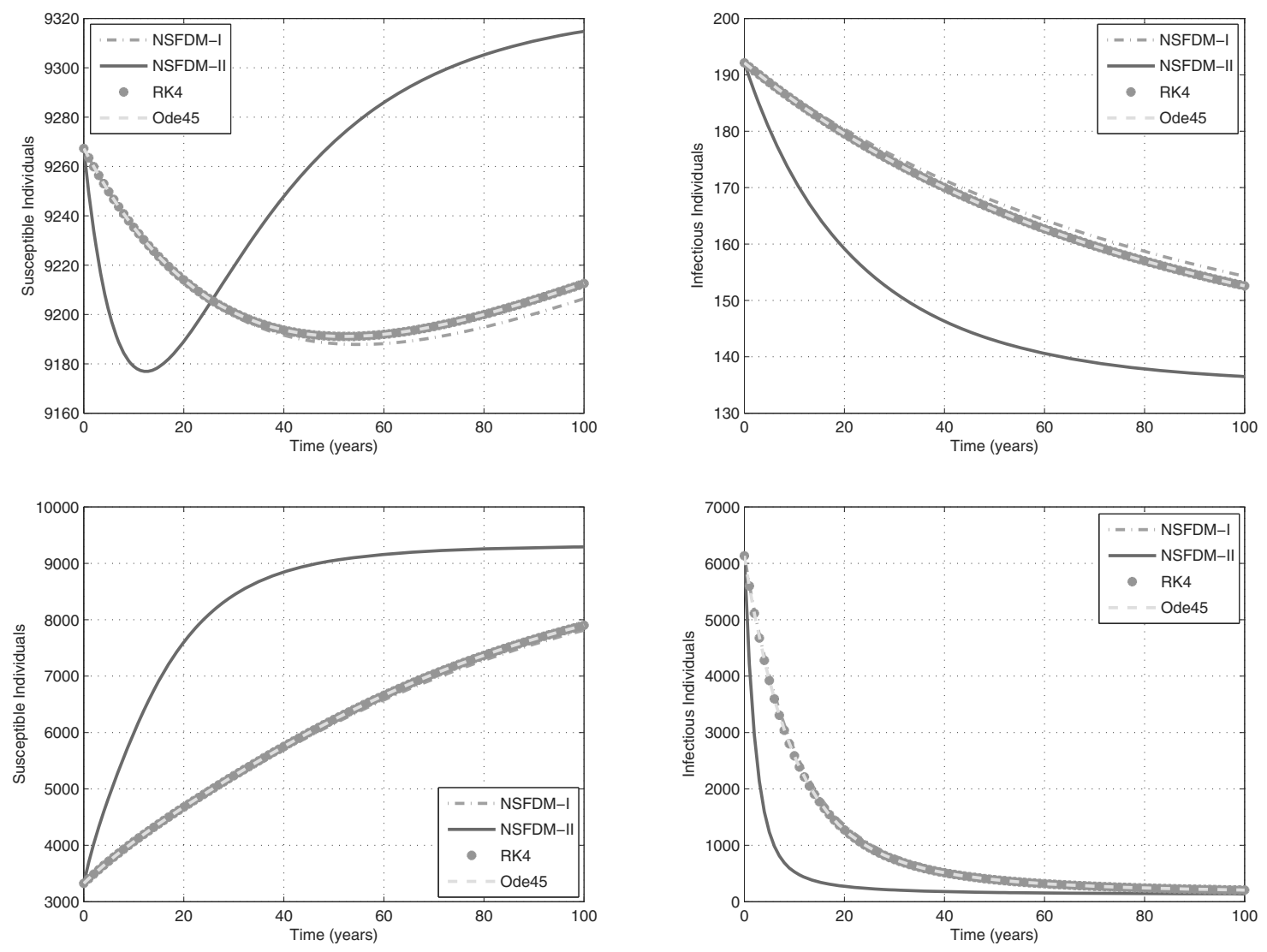

(a)

(b)

Fig. 7. Profiles of solutions (susceptibles $(S(t))$ (a) and infectious individuals $(I(t))$ (b)) obtained by using different numerical methods when $d=0.11$, with $\ell=1$. $(S(0), I(0))=(9267,192)$ (upper plots), $(S(0), I(0))=(3324,6135)$ (lower plots).

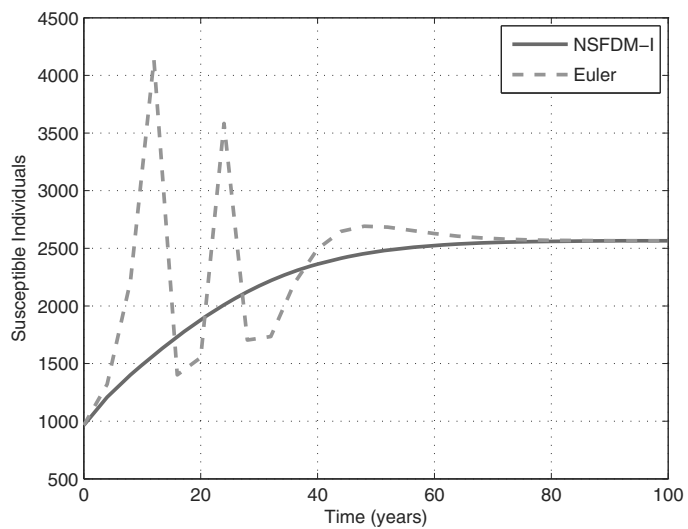

(a)

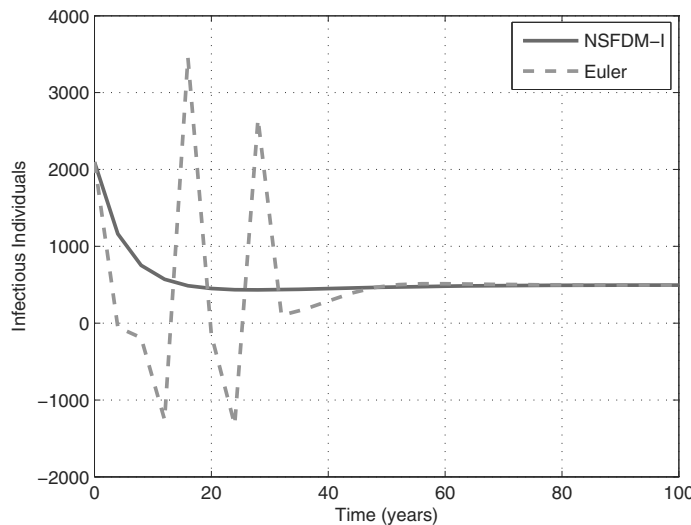

(b)

Fig. 8. Profiles of solutions (susceptibles $(S(t)$ (a) and infectious individuals $(I(t))$ (b)) obtained by NSFDM-I and the Euler method when $d=0.7, \mu_{2}=0.3$, with initial conditions $(S(0), I(0))=(965,2096) . \ell=4$.

by other numerical methods experience difficulties in either preserving the positivity of the solutions or in converging to the correct equilibria. Furthermore, since large step-sizes can be used, these methods save the computation time and memory.

It should be noted that, when numerical simulations 


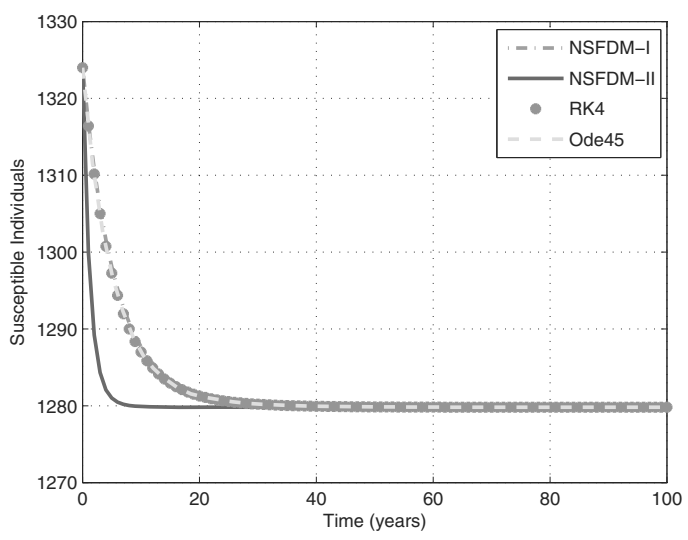

(a)

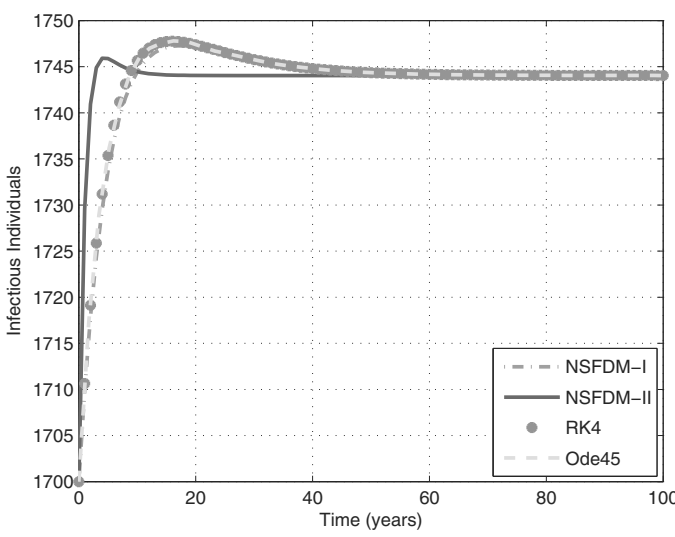

(b)

Fig. 9. Profiles of solutions (susceptibles $(S(t))$ (a) and infectious individuals $(I(t))$ (b)) obtained by using different numerical methods when $k=2$, with initial conditions $(S(0), I(0))=(1324,1700) \cdot \ell=1$.

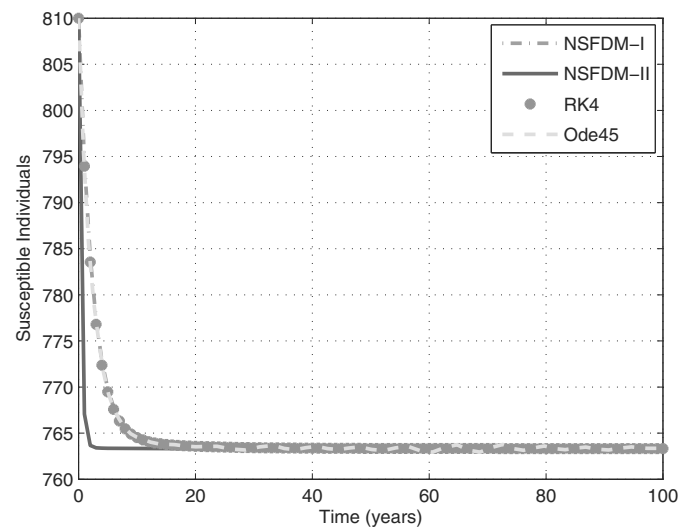

(a)

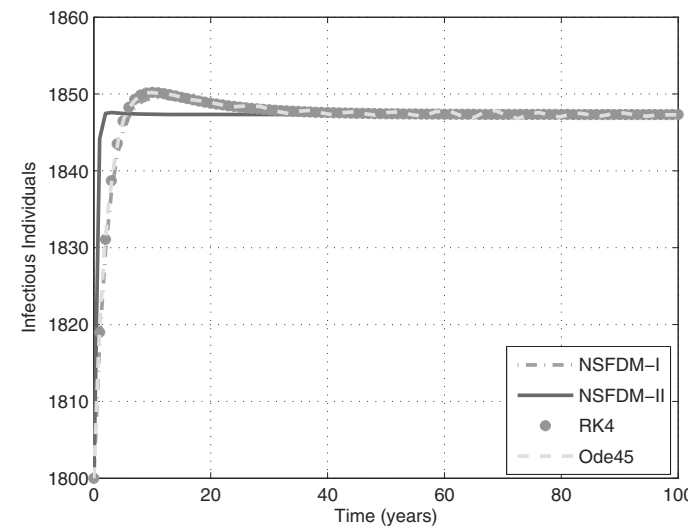

(b)

Fig. 10. Profiles of solutions (susceptibles $(S(t))$ (a) and infectious individuals $(I(t))$ (b)) obtained by using different numerical methods when $k=5$, with initial conditions $(S(0), I(0))=(810,1800) \cdot \ell=1$.

Table 10. Results obtained by different numerical methods for $k=10$ with initial conditions $(S(0), I(0))=$ $(500,1918)$ and different step-sizes. In this case, the endemic equilibrium is given by $\left(S^{*}, I^{*}\right)=$ $(523,1895)$.

\begin{tabular}{|l|l|l|l|l|}
\hline \hline$\ell$ & ode45 & RK4 & NSFDM-I & NSFDM-II \\
\hline 0.01 & Converge & Converge & Converge & Converge \\
1 & Converge & Converge & Converge & Converge \\
3 & Failed & Converge & Converge & Converge \\
4 & Failed & Converge & Converge & Converge \\
6 & Failed & Diverge & Converge & Converge \\
10 & Failed & Diverge & Converge & Converge \\
15 & Failed & Diverge & Converge & Converge \\
20 & Failed & Diverge & Converge & Converge \\
\hline
\end{tabular}

using a particular method are performed for a set of parameters that usually fit the model well, then the method normally tends to converge. However, a slight change in the values of these parameters can make some methods unreliable. In reality, one might expect (with a very little probability) some situations, for example, disease outbreaks in a community, when at a particular time there may be more infectious individuals than susceptibles. To test whether the numerical methods capture these dynamics, we have provided some more numerical simulations (see Tables 6, 7 and Figs. 3 and7 It is clear from these results that NSFDMs could mimic the relevant dynamics whereas the other numerical methods failed to do so.

Currently, we are investigating the applicability of the proposed method to solve mathematical models of HIV-TB co-infections. 


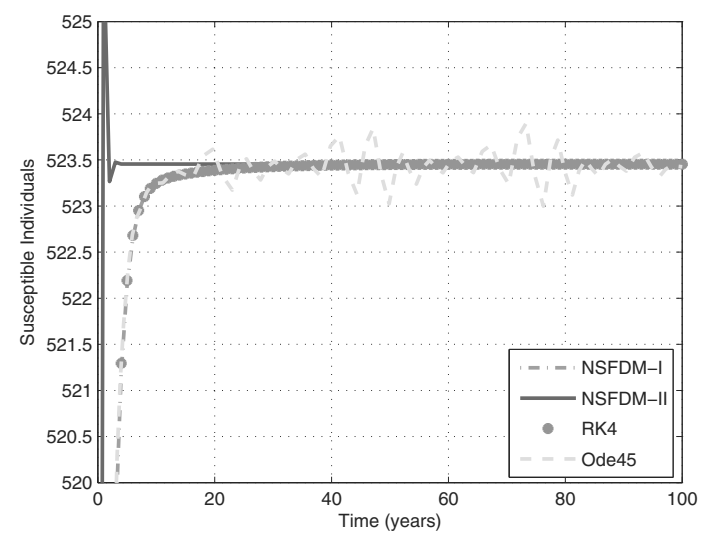

(a)

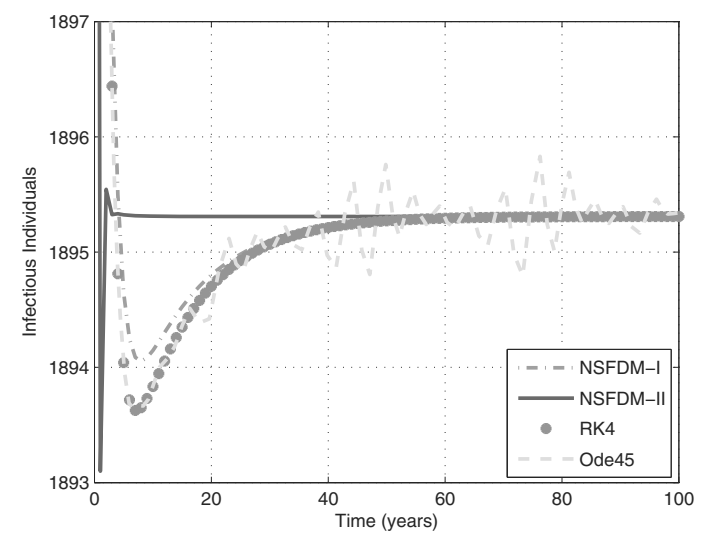

(b)

Fig. 11. Profiles of solutions (susceptibles $(S(t)$ (a) and infectious individuals $(I(t))$ (b)) obtained by using different numerical methods when $k=10$, with initial conditions $(S(0), I(0))=(500,1918) \cdot \ell=1$.

\section{Acknowledgment}

The first author acknowledges the Faculty of Mathematical Sciences, University of Khartoum, Sudan, for the financial support. The research of the third author was also supported by the South African National Research Foundation. We are grateful to all anonymous referees for their valuable comments and suggestions which helped us improve the presentation of this work.

\section{References}

Allen, L. (2007). An Introduction to Mathematical Biology, Prentice-Hall, Englewood Cliffs, NJ.

Anguelov, R., Lubuma, J.-S. and Shillor, M. (2009). Dynamically consistent nonstandard finite difference schemes for continuous dynamical systems, Discrete and Continuous Dynamical Systems: Supplement 61: 34-43.

Arenas, A., No, J.M. and Cortés, J. (2008). Nonstandard numerical method for a mathematical model of RSV epidemiological transmission, Computers and Mathematics with Applications 56(3): 670-678.

Bacaer, N., Ouifki, R., Pretorius, C., Wood, R. and Williams, B. (2008). Modeling the joint epidemics of TB and HIV in a South African township, Journal of Mathematical Biology 57(4): 557-593.

Brauer, F. and Castillo-Chavez, C. (2001). Mathematical Models in Population Biology and Epidemiology, Springer-Verlag, New York, NY.

Dimitrov, D. and Kojouharov, H. (2005). Nonstandard finite-difference schemes for general two-dimensional autonomous dynamical systems, Applied Mathematics Letters 18(7): 769-774.

Dimitrov, D. and Kojouharov, H. (2006). Positive and elementary stable nonstandard numerical methods with applications to predator-prey models, Journal of Computational and Applied Mathematics 189(1): 98-108.
Dimitrov, D. and Kojouharov, H. (2007). Stability-preserving finite-difference methods for general multi-dimensional autonomous dynamical systems, International Journal of Numerical Analysis Modeling 4(2): 280-290.

Karcz-Dulęba, I. (2004). Asymptotic behaviour of a discrete dynamical system generated by a simple evolutionary process, International Journal of Applied Mathematics and Computer Science 14(1): 79-90.

Gumel, A., McCluskey, C. and van den Driessche, P. (2006). Mathematical study of a staged-progression HIV model with imperfect vaccine, Bulletin of Mathematical Biology 68(8): 2105-2128.

Gumel, A., Patidar, K. and Spiteri, R. (2005). Asymptotically consistent nonstandard finite difference methods for solving mathematical models arising in population biology, in R. Mickens (Ed.), Applications of Nonstandard Finite Difference Schemes, World Scientific, Singapore, pp. 385-421.

Ibijola, E., Ogunrinde, R. and Ade-Ibijola, O. (2008). On the theory and applications of new nonstandard finite difference methods for the solution of initial value problems in ordinary differential equations, Advances in Natural and Applied Sciences 2(3): 214-224.

Jódar, L., Villanueva, R., Arenas, A. and González, G. (2008). Nonstandard numerical methods for a mathematical model for influenza disease, Mathematics and Computers in Simulation 79(3): 622-633.

Kadalbajoo, M., Patidar, K. and Sharma, K. (2006). $\varepsilon$-uniformly convergent fitted methods for the numerical solution of the problems arising from singularly perturbed general DDEs, Applied Mathematics and Computation 182(1): 119-139.

Kouche, M. and Ainseba, B. (2010). A mathematical model of HIV-1 infection including the saturation effect of healthy cell proliferation, International Journal of Applied Mathematics and Computer Science 20(3): 601-612, DOI: 10.2478/v10006-010-0045-z. 
Lubuma, J.-S. and Patidar, K. (2006). Uniformly convergent non-standard finite difference methods for self-adjoint singular perturbation problems, Journal of Computational and Applied Mathematics 191(2): 229-238.

Lubuma, J.-S. and Patidar, K. (2007a). Non-standard methods for singularly perturbed problems possessing oscillatory/layer solutions, Applied Mathematics and Computation 187(2): 1147-1160.

Lubuma, J.-S. and Patidar, K. (2007b). Solving singularly perturbed advection reaction equation via non-standard finite difference methods, Mathematical Methods in the Applied Sciences 30(14): 1627-1637.

Lubuma, J.-S. and Patidar, K. (2007c). $\varepsilon$-uniform non-standard finite difference methods for singularly perturbed nonlinear boundary value problems, Advances in Mathematical Sciences and Applications 17(2): 651-665.

Mickens, R. (2007). Calculation of denominator functions for nonstandard finite difference schemes for differential equations satisfying a positivity condition, Numerical Methods for Partial Differential Equations 23(3): 672-691.

Mickens, R. and Ramadhani, I. (1994). Finite-difference schemes having the correct linear stability properties for all finite step-sizes III, Computer in Mathematics with Applications 27(4): 77-84.

Mickens, R. and Smith, A. (1990). Finite difference models of ordinary differential equations: Influence of denominator functions, Journal of the Franklin Institute 327(1): 143-145.

Munyakazi, J. and Patidar, K. (2010). Higher order numerical methods for singularly perturbed elliptic problems, Neural, Parallel \& Scientific Computations 18(1): 75-88.

Patidar, K. (2005). On the use of nonstandard finite difference methods, Journal of Difference Equations and Applications 11(8): 735-758.

Patidar, K. (2008). A robust fitted operator finite difference method for a two-parameter singular perturbation problem, Journal of Difference Equations and Applications 14(12): 1197-1214.

Patidar, K. and Sharma, K. (2006a). Uniformly convergent nonstandard finite difference methods for singularly perturbed differential difference equations with delay and advance, International Journal for Numerical Methods in Engineering 66(2): 272-296.

Patidar, K. and Sharma, K. (2006b). $\varepsilon$-uniformly convergent non-standard finite difference methods for singularly perturbed differential difference equations with small delay, Applied Mathematics and Computation 175(1): 864-890.

Rauh, A., Brill, M. and Günther, C. (2009). A novel interval arithmetic approach for solving differential-algebraic equations with ValEncIA-IVP, International Journal of Applied Mathematics and Computer Science 19(3): 381-397, DOI: 10.2478/v10006-009-0032-4.

Rauh, A., Minisini, J. and Hofer, E.P. (2009). Verification techniques for sensitivity analysis and design of controllers for nonlinear dynamic systems with uncertainties, International Journal of Applied Mathematics and Computer Science 19(3): 425-439, DOI: 10.2478/v10006-009-0035-1.

Villanueva, R., Arenas, A. and Gonzalez-Parra, G. (2008), A nonstandard dynamically consistent numerical scheme applied to obesity dynamics, Journal of Applied Mathematics, Article ID 640154, DOI: 10.1155/2008/640154.

Xu, C., Liao, M. and He, X. (2011). Stability and Hopf bifurcation analysis for a Lotka-Volterra predator-prey model with two delays, International Journal of Applied Mathematics and Computer Science 21(1): 97-107, DOI: 10.2478/v10006-011-0007-0.

Zhai, G. and Michel, A.M. (2004). Generalized practical stability analysis of discontinuous dynamical systems, International Journal of Applied Mathematics and Computer Science 14(1): 5-12.

Hasim Obaid obtained his M.Sc. in industrial and computational mathematics in 2003 from the University of Khartoum, Sudan, and his Ph.D. in 2011 from the University of the Western Cape, South Africa. His research interests include mathematical analysis of models arising in biology specially those describing HIV and tuberculosis diseases and developing some numerical methods to approximate the solutions of such biological models.

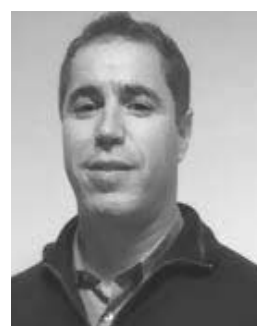

Rachid Ouifki is a senior researcher at the DST/NRF Centre of Excellence in Epidemiological Modelling and Analysis (SACEMA) at Stellenbosch University, South Africa. He holds a $\mathrm{Ph} . \mathrm{D}$. degree in applied mathematics. He is a coauthor of several papers and a regular reviewer for some scientific journals. He is also involved in several international projects. His current research includes ordinary, delayed and partial differential equations and their applications with a particular focus to bio-mathematics. He also works on optimal control theory and its applications, especially in the control of infectious diseases.

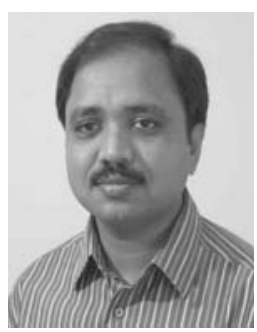

Kailash Patidar is a professor of applied mathematics at the University of the Western Cape, South Africa. His research is focused on numerical methods and scientific computing for the applied problems that arise from interactions between natural and life sciences as well as engineering. His research is a well balanced combination of analytical investigations and numerical experiments using finite difference, finite element and spline approximation methods. His current research involves the development of reliable numerical methods for mathematical models arising in population biology as well as those for option pricing problems in computational finance.

Received: 21 March 2012

Revised: 17 December 2012 